\title{
Forum
}

doi:10.1017/S037346331400071X

\section{Will Cost-Effectiveness Decisions Determine Future 4D Air Traffic Management Concepts?}

\author{
Peter Brooker \\ (Aviation Consultant) \\ (E-mail: p_brooker@btopenworld.com)
}

\begin{abstract}
Would an increased emphasis on cost-effectiveness and markedly reduced controller workload/costs determine the Four-Dimensional Air Traffic Management (4D ATM) Concept $-\mathrm{a}$ mindset change? Are there workable concepts that focus on flightpath conformance monitoring rather than a combination of conformance and hazard monitoring? Fundamental criteria for a conformance management-based system are identified to meet workload and cost goals. A 'Global Navigation Satellite System (GNSS)/Feedback Concept' is sketched, with radical ingredients to convert GNSS's accurate position fixes into accurate aircraft flightpath navigation. This eliminates air/ground trajectory synchronisation processing, and focuses conflict probing/planning tools on non-conforming flights. This concept would need to address key Human Factor concerns satisfactorily.
\end{abstract}

\section{KEY WORDS \\ 1. SESAR. 2. NextGen. 3. Air Safety. 4. GNSS.}

Submitted: 3 April 2014. Accepted: 22 September 2014. First published online: 7 November 2014.

1. INTRODUCTION. NextGen (USA's Next Generation Air Transportation System) and SESAR (Single European Sky ATM Research) are now familiar acronyms in the Air Traffic Management (ATM) community. Recent comprehensive official documents for these new generation ATM systems are JPDO (2011) and SESAR JU (2012). NextGen/SESAR's focus and nature have changed markedly over the decade of their development. Increasingly complex programmes have extended timescales, and many technical and operational issues still need Research and Development (R\&D). Airline bodies (IATA/AEA/ERA, 2013) and Government officials (e.g. Scovel, 2013) have been very critical. But this is not simply poor management. The problems are intrinsically extremely difficult: safety critical, new paradigm, large investment costs, multi-decision maker, multi-agent, etc. The purpose here is to show why and how a change in mindset, with an increased emphasis 
on cost-effectiveness, could shape the 'ultimate' form of the ATM system - the 4D ATM Concept - and help in driving the programmes forward.

This paper is largely standalone. However, it builds on the strategic benefits and costs analyses in Brooker (2012), and is a companion document to the technical description of a 4D ATM Concept discussed in Brooker (2014). The detailed analyses in those papers are not repeated here.

2. BACKGROUND. There are many ideas for further improving the ATM system, mostly incrementally, but also by complete rethinking of the operational concept. Many of the revolutionary ideas - to bring ATM into the information age' - were synthesised into a much more highly integrated system by Boeing (2001). The key elements were:

- All participants - flight crews, flight planners, ATC (air traffic control) system providers - accessing networked data for heightened collaboration, negotiation and strategic planning.

- Aircraft flying fuel-efficient 4D-space and time-trajectories, and up-to-date flight information being available on the intended path being accessible to all participants, accomplished by Flight Management Systems (FMS) incorporating autopilots, with (e.g.) effective Required Time of Arrival (RTA) facilities.

- Controllers safely managing much more traffic in larger sectors because they have strategic tools, with automation of routine and repetitive tasks.

- Innovative high-performing computing, communications, navigation and surveillance technologies.

Current NextGen/SESAR documents have updated the Boeing thinking, but the central points are much the same. The big development difference is that the fully integrated system is now the 'ultimate' goal, thus SESAR JU (2012): "The goal is a trajectory-based ATM system where partners optimise 'business and mission trajectories' through common 4D trajectory information and user defined priorities in the network." Compared with the Boeing ideas, there is much more emphasis on operational changes by a series of intermediate stages. However, the most noticeable difference is the timescale. Thus, the target for SESAR is now circa 2030, whereas Boeing suggested that a complete USA solution might be in place in seven years. Boeing was naïve about timescales for technological change in a safety critical and multi-stakeholder industry, but it did focus attention on the vital issue: the strategic commitment of stakeholders. The key stakeholders are the organisations that pay for investments in equipment and software, which are the government and the commercial airlines. There needs to be a compelling business case, returning valuable real cash outputs soon for money spent today.

Without huge financial incentives driving industry innovation to achieve the ultimate 4D ATM goal, the phased implementation of the necessary components is likely to be an extremely lengthy process, heavily constrained by investment cycles and step-by-step business cases. Progressive implementation of the enabling technological and operational steps might simply be halted because of the absence of assured medium term financial gains to stakeholders. Brooker (2012) identifies possible '4D killer apps'. Two key criteria are that the bulk of the benefits could not be obtainable 
through technologically 'cut down' non-4D-trajectory versions; and that they should not be largely dependent on particular future projections, e.g. very large traffic growth over a long period.

For government, the monetised social benefits of a 4D system to the public are such things as added growth in Gross Domestic Product (GDP) and employment, and passenger time-savings. These calculations are particularly relevant to business decisions if there are subsidies or grants - common in road/rail transport but much less so in the aviation transport sector. Potential large money gains of 4D identified in Brooker (2012) that are relevant to airlines - if not guaranteed killer apps - included:

- Fuel Efficiency - but will evolutionary changes deliver gains?

- Airspace capacity - but hugely traffic growth dependent, and will evolutionary improvements deliver gains?

- Airport capacity-dependent on (e.g.) very accurate time-keeping routinely achieved.

- Reduced navigational charges-dependent on major equipment costs and controller productivity gains.

Here, navigational charges are the charges made by ATM-mainly Air Traffic Control (ATC) services provision to airspace users.

3. ATM COSTS TO AIRLINES. A different way of looking at 4D benefits is to pose the question: "What specific kind of 4D ATM Concept will deliver the most financial gains to airspace users?" An investigation of potential financial gains has to start from the core features of present airline ATM-related costs. In Europe, ATC costs are raised through direct charges to users, mainly airlines. The USA currently relies on excise taxes on jet fuel and tickets plus federal budget support, but there has been pressure to change to a commercial basis charging users. IATA/AEA/ERA (2013) usefully notes that, for Europe in 2011, the air navigation services costs of ATM providers was $€ 8$ billion and system inefficiencies was $€ 5$ billion. The latter includes en route delays, route extensions, airport delays, arrivals holding and sequencing, and taxying costs. Many of these system inefficiencies are airport/schedule related airspace ATC issues. There are many ways of addressing system inefficiencies without recourse to 4D ATM Concepts, which could take out a significant slice of costs prior to full 4D operations, many of which have a long development history.

Table 1. Breakdown of European ATM/CNS provision costs in 2011 (PRU, 2013).

\begin{tabular}{lr}
\hline Category & Cost $(€ \mathrm{M})$ \\
\hline Staff & \\
$\quad$ Operational controllers & 2,360 \\
$\quad$ All others & 2,540 \\
Non-staff operating & 1,410 \\
Depreciation & 904 \\
Cost of capital & 562 \\
Exceptional Items & 63 \\
Total & 7,839 \\
\hline
\end{tabular}


Taking out large slices of the annual $€ 8$ billion costs is very attractive - and those reductions are for every year into the foreseeable future.

Table 1 breaks down the total (PRU, 2013). The dominant element is staff costs. About half of these are for operational controllers. The other staff costs are to keep the systems going (e.g. maintenance engineers), to develop the system (planning and project engineers), safety analysts, $R \& D$ workers, etc, as well as necessary management and service staff. These other costs are roughly in proportion to the number of operational controllers until economies of scale appear. Similarly, the various financial items will tend to increase roughly in line with controller numbers-again with the likelihood of some economies of scale when controller numbers are large. Thus:

\section{Total ATM cost $\sim \propto \quad$ Number of controllers}

The symbols $\sim \propto$ means 'roughly proportional'. Controller numbers in turn correlate with the number of airspace sectors they handle at peak demand times. Thus:

\section{Total ATM cost $\sim \propto$ Peak number of sectors}

Multiplying and dividing the right hand side by 'Peak aircraft count per sector' gives:

$$
\text { Total ATM cost } \sim \propto \frac{\text { Peak number of sectors } \times \text { Peak aircraft count per sector }}{\text { Peak aircraft count per sector }}
$$

The numerator now has a simple interpretation, hence:

$$
\text { Total ATM cost } \sim \propto \frac{\text { Peak aircraft count in whole airspace }}{\text { Peak aircraft count per sector }}
$$

Hence, for a given peak throughput in the whole airspace, the Total ATM costs are roughly inversely proportional to the 'Peak aircraft count per sector'. An exact calculation would involve several scaling factors but the key relationship would still be there. The peak aircraft count per sector is its capacity. It is the maximum number of aircraft that a controller can instantaneously handle while assuring safety and not risking excessive workload. (There are variant capacity definitions, e.g. focusing on the hourly throughput for a sector, but the general principles involved are the same.)

4. SECTOR CAPACITY IN NEXTGEN/SESAR. Correlating sector capacity to controller workload has been the subject of research for several decades. A current assessment is Welch et al. (2007). This models workload by summing the estimated load for four task types: background, transition, recurring, and conflict tasks.

Table 2 lists characteristics of the four types. The total workload intensity $\mathrm{G}$ is the sum of the task times devoted to each of the events modelled in these task types. Each varies in a different way with the amount of traffic in the sector. Figure 1 illustrates these variations for a typical sector, with the total workload intensity compared with the Workload Limit for the sector - setting the sector capacity at an aircraft count of about 16 . The approximately quadratic Conflict term quickly dominates the other contributions.

SESAR documents typically set a goal of a 3-fold increase in capacity values and simultaneously at least a halving of ATM provider costs per flight. Such a tripling in 
Table 2. Controller task types- after Welch et al. (2007).

Background: routine activities - configuring displays, coordinating with managers and supervisors, maintaining work areas, verifying surveillance performance, examining weather forecasts, etc

Transition: when aircraft passes through the sector, including hand-off acceptance, initial contact, familiarization with flight plan information, and initial route planning

Recurring: includes in-sector activities such as traffic scanning, restricted airspace and hazardous weather avoidance, flight plan changes, and status updates, conformance monitoring and separation planning

Conflict: includes conflict detection, vectoring for conflict resolution, consideration of secondary encounters, and post-conflict route recovery

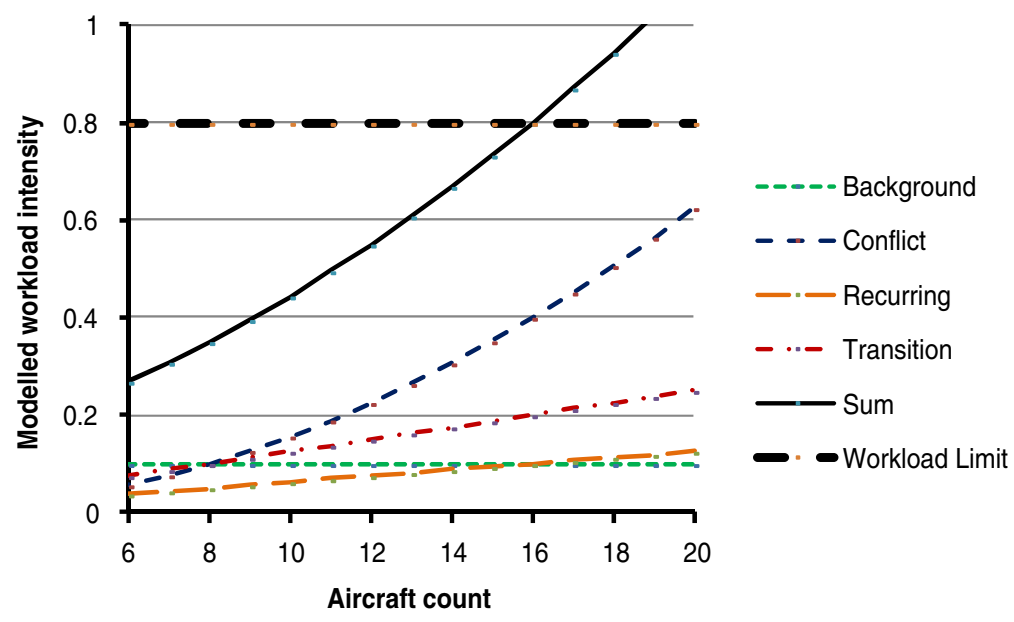

Figure 1. Modelled workload intensity versus aircraft count for a sector volume of $10,000 \mathrm{Nm}^{3}$ - after Welch et al. (2007).

capacity would presumably imply meeting roughly 'times 3' traffic demand in every sector, so the Conflict workload in Figure 1's standard sector, with its quadratic variation, would increase by a factor of around $3^{2}=9$. If costs per flight are to be halved then this implies a total cost for handling 'times 3 ' traffic would be 'times $1 \frac{1}{2}$ ' the current costs - and hence 'times $1 \frac{1}{2}$ ' controllers would handle a standard sector. These numbers are obviously very crude indicators, but they are consistent with the targets and current cost structure. The simple conclusion of these coarse sums are that the total costs to users - and numbers of controllers - would still tend to increase markedly, albeit not as fast as traffic demand. Airlines would surely be much more committed to an ATM Concept that delivers higher capacity and reduced future total costs.

Many hundreds of research papers examine different aspects of changes to controller tasks, much of it relating to NextGen/SESAR. This indicates healthy debate, but implies that there is little agreement on a clear 'winner' 4D ATM Concept. Present public documents do not present clear pictures of the 4D ATM Concept goal, with many technical/operational options still being open. As noted, this is in major part because the ATM system operation is seen as building on a succession of innovatory steps. Unfortunately, this leads to generalities, e.g. SESAR JU (2012): 
"The SESAR Concept of operations will drive changes to the procedures being used by all stakeholders, and in particular will start to redistribute responsibilities as defined in current procedures between technology, controllers and flight crew."

There is a strong awareness of many high-priority risk areas, e.g. (SESAR JU, 2012): "Human Factors (HF) not integrated in Concepts, development and validation (with operational staff), including applying minimal standards and unrealistic assumptions (especially human workload and automation)."

The open questions and already identified risks mean that there is not a high 'NextGen/SESAR ownership' by major stakeholders. The large benefits may well be there but it is not clear which crucial ATM system changes will deliver them (Scovel, 2013). Is there some form of 4D ATM Concept that would potentially motivate major stakeholders: a safe high-performing system but with much lower operational costs, and not dependent on completely novel technology or software developments?

\section{INCREASED SECTOR CAPACITY IN 4D ATM CONCEPTS?}

How can sector capacity values be safely (i.e. building on existing safety defensive layers), substantially and cost-effectively increased by a 4D ATM Concept? Will existing development programmes starting from the present system yield the large improvements desired by customers? The fact that a 4D ATM Concept necessarily uses an agreed - and conflict free - flightpath contract between the flight and ATM offers another option for system operation. The crux is the distinction between the two types of safety intervention function: Conformance Management and Hazard Management. This difference in controller roles is clearly set out in Simpson and Ausrotas (1991):

- Conformance Management (CM) is an intervention process that attempts to ensure that each aircraft conforms to its assigned path. It assumes each aircraft has an assigned conflict-free path...

- Hazard Management (HM) is an intervention process that attempts to ensure that there is safe separation between all pairs of aircraft. It makes a recurrent projection of the actual paths of aircraft. If this projection indicates that a hazardous encounter is likely, it is concerned with finding a safe resolution of the encounter...

(Reynolds and Hansman (2003) is an important in-depth examination of Conformance Management characteristics.) The key point here is that the focus of Hazard Management is on potentially conflicting pairs of flights, while Conformance Management is simply about flights themselves. Can the need for 'quadratic function' Hazard Management be eliminated for normal operations? Present R\&D mainly focuses on reducing the size of the quadratic coefficient for conflict-related tasks, and these have already led to successful operational implementations, e.g. iFACTS (Rolfe, 2014).

A system based on Conformance Management requires several ingredients. Figure 2 shows schematic snapshots from a controller's main display in a 4D era (which would be supplemented by problem-focused displays). It shows three aircraft flightpaths - A, B and C. Overlaid on each flightpath are three aircraft position snapshots: times 1, 2, and 3. A small filled circle symbol shows the actual position 


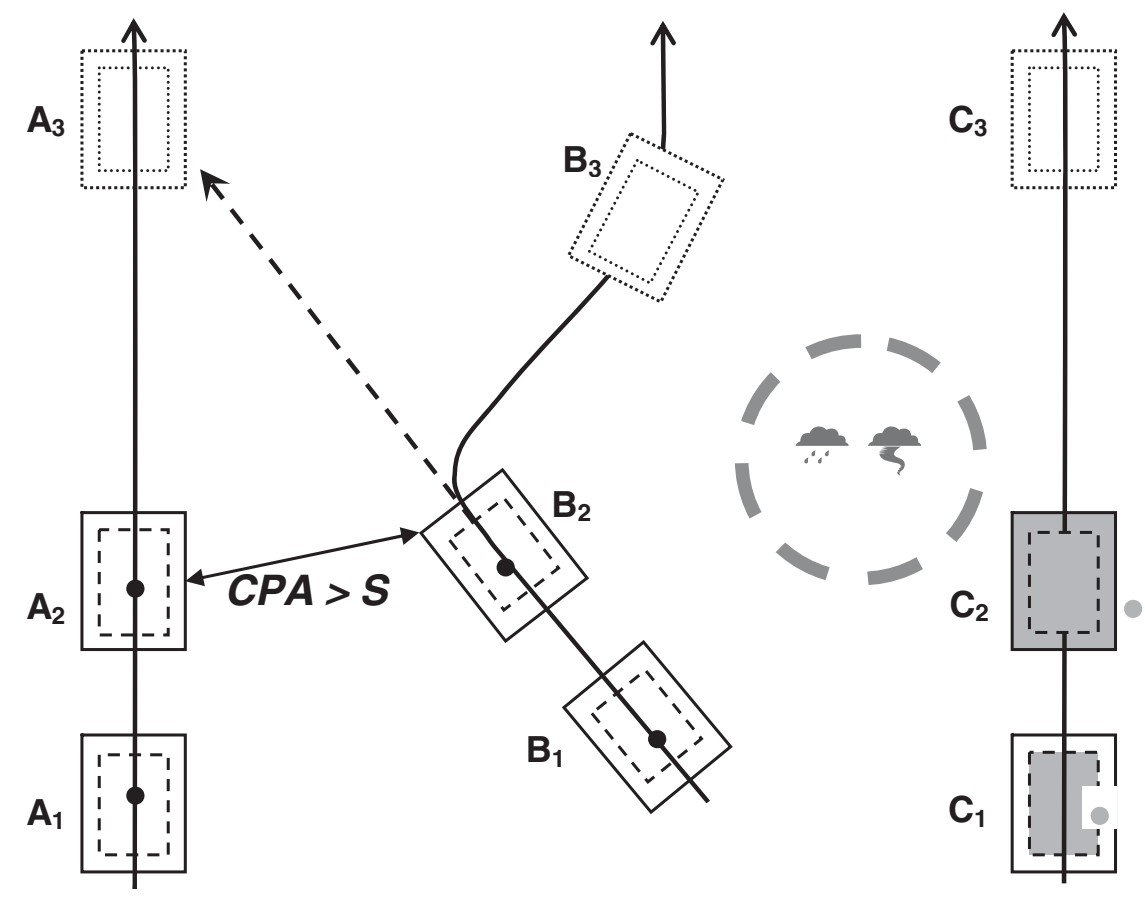

Figure 2. Conformance Management and Hazard Management issues in 4D ATM Concept shows overlays of the main display at a sequence of times 1, 2, and 3. Small filled circle - reported aircraft position. Rectangles - warning and conflict boxes positions.

of the aircraft at the first two times. The planned position of the aircraft is at the centre of a conformance box - the 'contract box'-whose size is determined by aircraft navigation, flight performance etc. For safety, the aircraft symbols and boxes must of course be accurately displayed. Each flightpath time shows two boxes in plan view for each flightpath time (the boxes are three-dimensional but vertical navigation is not discussed here). The larger one has dimensions that an on-flightpath aircraft should hardly ever go outside; the smaller - 'warning box' - dimensions are measures of the extremes of typical navigation, and indicate to the controller that there might possibly be an issue to resolve. Time 3 boxes show where the aircraft should be at that time according to the contracted flightpath. CPA is the Closest Point of Approach, showing where aircraft $\mathrm{A}$ and $\mathrm{B}$ are nearest. These planned contract box separations must always be greater than the separation minimum for this airspace ' $\mathrm{S}$ ', i.e. conflict-free. $\mathrm{S}$ is in the main determined by the ability of controllers to deal with gross deviations from flightpath.

Aircraft A is on a simple straight-line course. Aircraft B turns right just at about time 2 in order to avoid very bad weather, shown by some cloud symbols in a circle. With current ATM, a controller might well judge that aircraft B poses a potential risk to aircraft $\mathrm{A}$, as the track extrapolation from times 1 and 2 projects to a possible collision at time 3 . But if aircraft B keeps to its contracted flightpath, there would be no risk of an accident. The only way a collision can result from aircraft verified as being in an accurate box is from a non-conforming excursion. The difference between the present system and this $4 \mathrm{D}$ version is that the ATM has full information 
of future flight intent for the aircraft under control. In contrast, aircraft B and C - the extrapolated paths from times 1 to 2 not breaching the separation minimum - would probably not be shown as potentially conflicting in the present system, but in reality there could be a high risk of an incident. Aircraft $\mathrm{C}$ is markedly not adhering to its contracted flightpath: at time 1 it has already breached the warning box, and by time 2 it is outside the contract box. The controller would be warned about these infractions by (say) flashing symbols (akin to the present-day conflict alerts) - shown as grey fills here. $\mathrm{He} / \mathrm{she}$ would then have to act decisively to ensure that the nature of the problem was understood and that the aircraft was re-planned on to a conflict-free new flightpath and the other 'system actors' are consulted/informed.

This description of a 4D era in Figure 2 is extremely important in terms of controller workload. It shows the difference between a system relying on conformance management and one relying on a combination of conformance management and hazard management. With a trusted conformance-based system and planned conflictfree flightpaths, the controller does not have to project forward all the relevant combinations of future aircraft flightpaths, either mentally or with a conflict-probing tool - as long as the aircraft is within the contract box. Controllers must trust the system to indicate the best predictive estimate of its future position - the contracted 4D flightpath. The system might well highlight to the controller if an extrapolated position is in some sense a major test of conformance, so that he/she would check that aircraft B's sharp manoeuvre is actually taking place to plan. This conformance managementbased system would eliminate the quadratic growth term in workload in Figure 1, and, given the task workload reductions in the other workload types, it could generate much higher sector throughput at acceptable workload. Note that the current quadratic term mainly corresponds to controllers searching for potential conflicts rather than them dealing with actual conflicts.

Proving that a 4D ATM system is 'acceptably safe'-e.g. with mid-air collision risk some degree better than the present system - would need detailed analysis, as would the security aspects of the information processing. The key point in Figure 2 is that the aircraft symbol has to appear within the warning/contract boxes - a pivotal safety event that might evolve to a potential collision scenario. A collision could also occur if some kind of failed initiating event meant that the contract box is not successfully initialised, e.g. if an aircraft Mode $\mathrm{S}$ transponder failed or were to be switched off.

\section{MEETING CONTROLLER WORKLOAD AND COST GOALS.}

The following list tries to identify the fundamental criteria for a conformance management-based system to assure that the controller workload and cost goals discussed above are met:

- The technical ATM, aircraft and infrastructure systems have to demonstrate extremely high reliability and performance. Controllers and pilots have to be able to trust the systems - and each other - should action be required. If trust is lacking then the response might well be large numbers of extra checks and verifications, with workload implications.

- Contract/warning boxes have to have small dimensions, e.g. in comparison with the separation minimum applied. Otherwise, there is a very inefficient use 
of airspace, with the planning of flightpaths in densely occupied airspace potentially being heavily constrained by existing contracted flights.

- The frequency of warning/contract box excursions when aircraft are navigating 'normally' must be very small. Otherwise, controllers will have to spend excessive amounts of time checking that aircraft are actually on the agreed flightpath, using 'Decision Support Tools'. Safety and workload considerations mean that multiple warnings in close time proximity are highly undesirable, in particular if they occur in a particular region of sector airspace.

- Ground automation/trajectory generation must be synchronized and compatible to a high degree with aircraft automation/trajectory generation using inter alia FMS with autopilots. Current R\&D focuses on a largely automatic process of data interchange between air and ground systems. "The ATM system relies on all actors having the same view" (ICAO, 2012); "This represents a significant challenge to both ground operations and flight deck operations" (NextGen Institute, 2012). This synchronization must be in place for every instant of the flight.

How close are NextGen/SESAR to specifying a 4D ATM system that meets these criteria? The continued R\&D into trajectory prediction has generated hundreds of research papers. Unfortunately, industry experts say: "The large-scale interactions, complexity to decision-support tool capabilities for multiple, complex states of trajectories, etc., have not been clearly addressed or rationalized. The simple examples clearly show that the assessment of the dynamic aspect of trajectory operations is really hard. However, we are really not much closer to the solution..." (NextGen Institute, 2012).

7. GNSS/FEEDBACK CONCEPT SKETCH. Is there a variant 4D system Concept - i.e. making some significant changes to current R\&D thinking - that could more readily meet these four necessary criteria? Any significant weaknesses in reliability or performance introduce workload but also, and much more important, safety issues. Normal aircraft navigation must be extremely accurate everywhere in the controller's sector, so that warning and contract boxes sizes can be set at several standard deviations but still be 'small'. Aircraft Flight Management Systems (FMS) and autopilots already deliver highly accurate lateral ('LNAV') and vertical ('VNAV') navigation modes, but longitudinal navigation can be considerably hampered by errors in forecast wind. How can this along-track accuracy problem be solved? The final criterion is the subject of much of the current R\&D work. Ground predictions of the aircraft flightpath have essentially to simulate very accurately the operation of the aircraft's FMS, autopilot etc, taking into account the wind profile, the weight, and the performance characteristics of individual aircraft types.

Two radical strategic ingredients might enable the criteria to be satisfied, noting that Section 9 covers some tactical modifications and revision mechanisms:

- The flightpath to be used is that programmed by the aircraft FMS with forecast wind for each of the flight segments. The positional data for every time on this flightpath is transmitted to ATC in advance, either as positions at each instant or as accurate mark-up tables describing position/time evolution during each 


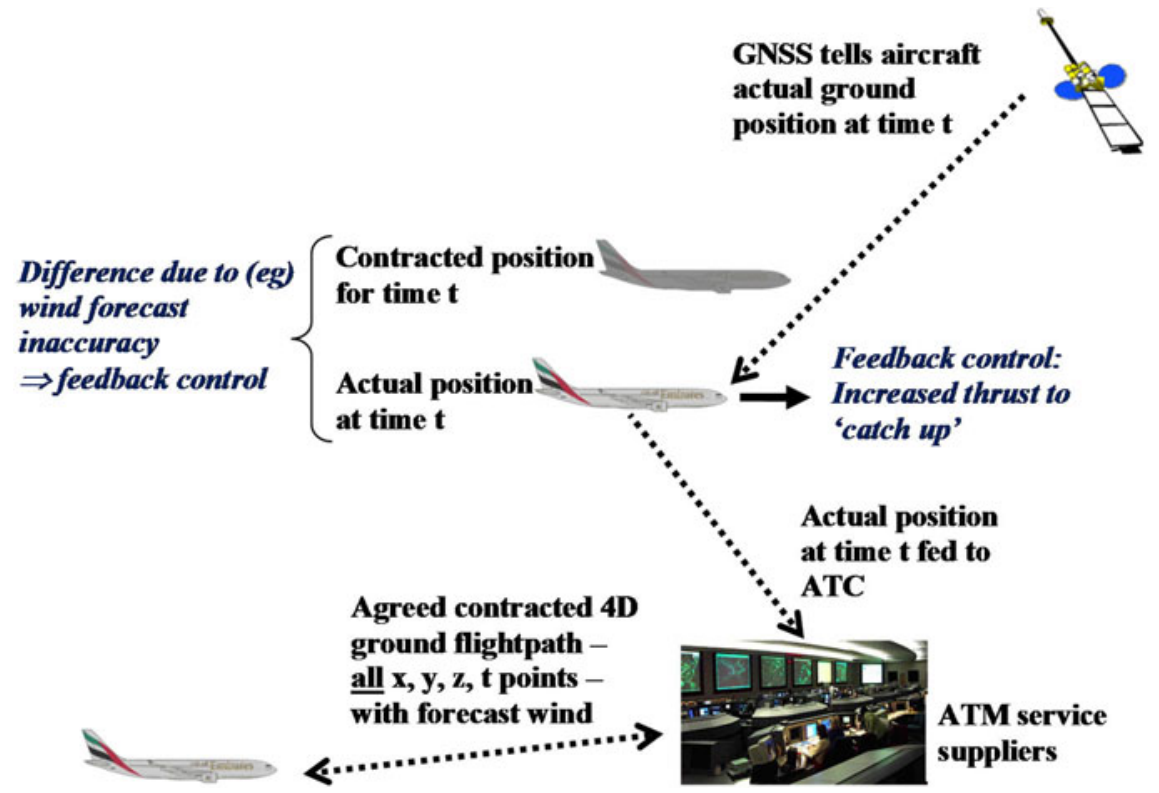

Figure 3. GNSS/feedback system.

flight segment. [NB: wind forecasts are becoming increasingly accurate, e.g. see the Met. Office (2014) figures for $250 \mathrm{hPa}$. Improved wind forecasting is recognised as having fundamental importance to NextGen, e.g. Reynolds et al., 2013).]

- The aircraft must fly the agreed ground track accurately. This is achieved by a navigational feedback control loop, with flightpath errors determined by Global Navigation Satellite System (GNSS) position being used to modify engine thrust and control surfaces.

Figure 3 sketches this GNSS/Feedback Concept: Brooker (2014) provides some technical details. It shows an aircraft that is 'late' at its planned x, y, z, position at time $t$ because of a small error in the forecast wind. This is detected by the difference in the planned position for that time and that measured by GNSS. Appropriate corrections are made to thrust setting and control surfaces. These corrections would be determined by a tailored feedback control system, akin to that used in automobile cruise control. The key points here are that the second and third criteria at the beginning of Section 6 can be satisfied because of GNSS precision and the removal of environmental factors, in particular wind errors; and that the final criterion is trivially satisfied because air and ground are using the same flightpath trajectory - so a synchronisation process is not required. (There is some similarity in concept with modern FMSs using the difference between the expected and sensed winds to define a wind correction term, which is "blended" with the wind estimates for future segments.)

The key innovation is that GNSS's accurate position fixes are 'transformed' into accurate aircraft flightpath navigation. There are two major gains in terms of system design: taking out air/ground trajectory synchronisation processing and removing the need for ground trajectory prediction for conforming traffic. The controller's use of 
conflict probing/planning tools is focused on non-conforming flights. The changed Concept has a price: extra FMS/datalink communications functionality. However, note that the FMS changes would be accessing computed data, rather than revising the core aerodynamic calculations. Some more details of the technical issues and potential problems - mainly requiring modelling and estimation - with such a system are examined in Brooker (2014). It should also be noted that any 4D ATM Concept has to deal with transitional problems, i.e. when a sizeable proportion of aircraft would not be 4D-capable. Controller tools and airspace arrangements would have to ensure the safety of all 4D and non-4D combinations of aircraft pairs. The GNSS/ Feedback Concept would however be able to handle conforming 4D-equipped flights with much reduced workload, as they do not themselves generate conflicts in normal operations.

8. HUMAN FACTORS AND GNSS/FEEDBACK CONCEPT. 4D ATM systems need specific controller roles and tasks. As noted earlier, HF is identified as a major problem area. Unfortunately, the NextGen/SESAR literature does not explain what precise changes these would involve in practice. Thus, from SESAR JU (2012):

"Controllers and flight crew will face a significant amount of change relating to the Essential Operational Changes... Controllers will have to face the new trajectory Paradigm... Such an evolution must be reviewed to guarantee that their expertise is maintained and their tasks are appropriately structured in all situations."

But 'people' aspects are not second order automation issues, somehow to be 'worked around'. The redesign of controller jobs must ensure that the system is genuinely human-centred and that they can/will use new skills effectively. Theoretical analyses of controller workload are meaningless if people cannot actually make the new system work safely and successfully. Would the GNSS/Feedback ATM system have fatal HF weaknesses? This and the next Section examine these vital safety questions.

There have been several efforts to examine the HF impact of changes to controller roles and responsibilities resulting from radically different ATM Concepts. Changes need to be considered in two contexts: normal and abnormal operations. Figure 2 shows aircraft $\mathrm{A}$ and $\mathrm{B}$ in normal operations, but aircraft $\mathrm{C}$ is definitely an abnormal flightpath. However, abnormal operations potentially cover many other things, e.g. incursion by rogue flights, pilot/controller communications breakdown. Normal operations correspond to the capacity of the system - the focus here - and the creation of/responses to abnormal operations determine system safety.

A very useful review of HF in future ATM is Langan-Fox et al. (2009). This focuses on five key HF factors: situational awareness, workload, trust, stress, and boredom. The second and third of these are discussed briefly above; the last two have (roughly) their day-to-day meanings. Situational awareness needs a brief explanation. A controller gets information from several sources - the screen, data displays, voice exchanges with pilots/colleagues, etc-and from these creates a mental picture that should pick out all the important features of what is happening: Situation Awareness (SA). The situation awareness term originated in military pilot studies, with a simple definition (Adam, 1993) being “Knowing what's going on so you can figure out what 
to do." In civil applications, Endsley (1995) identifies three levels of failure associated with reduced SA:

- Level 1 -Failure to correctly perceive information.

- Level 2 -Failure to correctly integrate or comprehend information.

- Level 3 -Failure to project future actions or state of the system.

The GNSS/Feedback Concept would assist SA at all three of these levels. If some abnormal things occur, a controller with a Level 3 failure might make decisions that put aircraft at risk because he/she is making decisions based on an imperfect mental picture. But good SA does not guarantee good decisions. And how good does a controller's mental picture have to be in order to be viewed as acceptable?

Langan-Fox et al. (2009) focuses on HF factor measurement. This is vital: if a factor can be reliably measured then it is possible to set acceptability bounds on its operational usage, e.g. the workload limit in Figure 1. The Langan-Fox et al. (2009) literature review suggests that there are useful, if not completely definitive, examples of measurements for all the key factors in ATM contexts, except for boredom. Boredom is often coupled with vigilance tasks and the experience of monotony. Controllers may be expected to suffer from monotony if their job mainly entails vigilance tasks coupled with traffic flows that are uneventful and largely repetitive. Straussberger (2006) is generally acknowledged as the main - almost sole-source on this topic.

Controllers are responsible for the process, initially registering aircraft flightpaths and validating the boxes, but could be viewed as acting as 'passive operators' during the passage through the sector. The 'cognitive task demands' could be much less than at present, in particular by the elimination of 'generation' tasks, as when controllers currently construct plans for safe flightpaths. Might controllers increasingly lose SA and become deskilled in traditional control tasks; and hence be less able to take appropriate corrective action in the event of (e.g.) equipment malfunction? These are thus real safety issues, but there are also important industrial relations issues as controllers react negatively to the combinations of (e.g.) vigilance and potential deskilling. The GNSS feedback 4D ATM Concept would obviously be susceptible to these HF concerns - current NextGen/SESAR work tends to involve controller involvement with conflict probing tools in normal operations.

However, there are several positive avenues to ensure that the controller has a mixture of vigilance and active tasks. First, it is not inevitable that controller performance will be worse in more automated systems - e.g. see Wickens et al. (2010). Second, these kinds of issue are not new to aviation-or indeed to other safety critical industries. When flight deck automation was introduced, there were concerns about the transfer of safety-critical functions 'away from pilots', and increasingly abstract connections with the aircraft's systems. There have been continuing efforts to improve performance in this area, e.g. PARC/CAST Flight Deck Automation WG (2013).

9. CONTROLLER PROBLEMS \& TACTICAL TASKS. Figure 2, with a normal operation the great bulk of the time and infrequent contract box excursions, paints an incomplete picture. In reality, the Concept cannot be a Procrustean affair: controllers must be able to deal with immediate problems and 


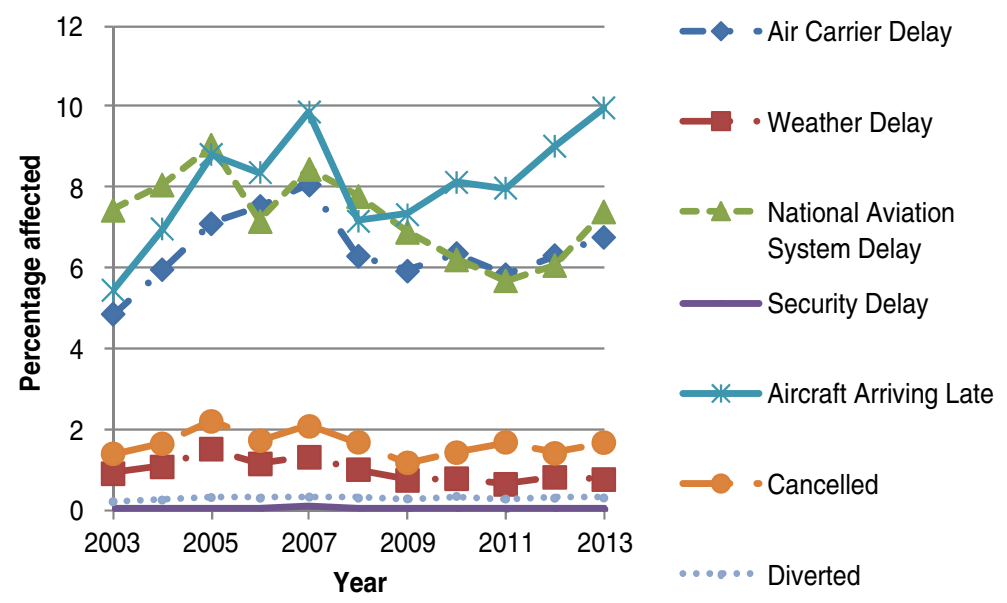

Figure 4. USA Arrivals Performance-July figures (RITA, 2014). See source for detailed definitions of the terms.

make tactical changes to deliver operational benefits. Three classes of change would generally require a renegotiated conflict-free flightpath for the flight's remaining segments:

- As noted, controllers must deal tactically-dependent on the precise circumstances - with all safety-related events, arising from aircraft failing to keep within the contract box.

- Revisions will be needed if the actual wind differs markedly from the forecast wind, leading to excessive fuel usage.

- The task of dealing with aircraft whose 4D contract needs to be revised because of aircraft delays, the need to schedule airport runways efficiently, changes in constraints, etc.

The first class should be very infrequent. The second class would generally arise from speed increases rather than speed reductions. The frequency of these changes would be less when wind forecasts are very accurate. Note that the intended fuel-efficient flightpath is not 'an ideal optimum' - it is constrained by the contracted times at waypoints/fixes and at the destination, potential closest approaches to other flights, etc.

Some current data helps to illustrate the possible impact of the third class on workload. Figure 4 (RITA, 2014) shows USA statistics on the percentage of arriving flights that were not on-time in July (a peak month) of the last ten years - European data shows broadly similar patterns. It is broken down into delay/cancellation categories. As noted on the Figure, these categories conceal several subtleties, e.g. regarding weather. The key point about Figure 4 is that delays/cancellations of all kinds currently affect about a quarter of operations. In a 4D environment, the percentage breakdown would be expected to be very different, with presumably fewer weather-related and ATC-provider delays (given continued forecasting and organisational performance improvements), but the size of the changes is not easy to estimate. The airport 4D time contracts in such a system would presumably be much 
tighter than existing scheduling tolerances, in order to make highly effective use of runway capacity, so there would be less tolerance of delay. En route delays arising from sector capacity constraints would be expected to be less frequent, assuming proper controller allocation. Whatever the nature of the changes, it would be necessary to modify a proportion of aircraft contracted flightpaths in order to update airport slots/schedules. There would be a sequence of modifications and substitutions.

Estimating the workload contribution from modifications to flightpaths has to be speculative. For a tightly structured 4D system, there is no obvious immediate relationship between the extent of delays and the number of controller flightpath interventions that would be required. Suppose a controller is handling 32 flights at a particular time and that $25 \%$ of flightpaths need modification. Thus, eight of those flights are modified. However, if flights were to pass through a typical (current) airspace sector in about 20 minutes (Welch et al., 2007), with (say) four sectors per flight, on average the sector controller would have to carry out the confirmation process for two modified flights out of the 32, and handle any remaining transitional flightpaths arising from earlier changes. To carry out these functions requires the controller's tasks to be linked to Airline Operations Centres (AOC) and airport operations in a much more coordinated fashion than at present. The design of effective flight information processing and decision-making systems involving aircrew, en route/airport control and AOCs in a trajectory-based environment is a major task.

The three kinds of renegotiations would have to occur in any kind of NextGen/ SESAR concept. There would be differences, of course. For example, large wind errors may make it possible to comply with RTA constraints only at the expense of significant extra fuel burn (Reynolds et al., 2013). Would there be markedly higher fuel or other operational penalties for a Concept focusing on Conformance Management than current NextGen/SESAR proposals?

10. CONCLUSIONS. Would an increased emphasis on cost-effectiveness and markedly reduced controller workload/costs determine the 4D ATM Concept - a mindset change? Are there workable Concepts that focus on flightpath conformance monitoring rather than a combination of conformance and hazard monitoring? Fundamental criteria are identified for a conformance management-based system that would meet reduced controller workload and cost goals. A radical 'GNSS/Feedback Concept' would 'transform' GNSS's accurate position fixes into accurate aircraft flightpath navigation. This would eliminate air/ground trajectory synchronisation processing, and focus conflict probing/planning tools on non-conforming traffic. There are technical issues to resolve, and this Concept would need to address key Human Factor concerns.

NextGen/SESAR may well develop smoothly and cost-effectively to an ATM Concept reliant on contracted 4D flightpaths. The Concept roughly sketched here, focusing on controller Conformance Management, is a radical variant that might attract much more stakeholder support because of its stress on large ATC provision cost-savings. If options targeted at contracted 4D flightpaths do not secure long-term stakeholder support, the most likely future scenario is continued evolutionary developments based on computer assistance. 


\section{ACKNOWLEDGEMENTS}

A preliminary version of this paper was presented to the Royal Institute of Navigation's 2014 Aviation Conference. I would like to thank participants for critical comments, which I have attempted to address. I would also like to thank one of the reviewers for highlighting material that could be further explained.

\section{REFERENCES}

Adam, E. C. (1993). Fighter cockpits of the future. Proceedings of $12^{\text {th }}$ DASC, IEEE/AIAA Digital Avionics Systems Conference, 318-323.

Boeing (2001). Air Traffic Management Revolutionary Concepts That Enable Air Traffic Growth While Cutting Delays.

Brooker, P. (2012). 4D-Trajectory Air Traffic Management: Are There 'Killer Apps?' Parts 1 and 2. The Journal of Navigation, 65(3), 397-408; 65(4), 571-587.

Brooker, P. (2014). A 4D ATM Trajectory Concept Integrating GNSS and FMS? The Journal of Navigation, 67(4), 617-631.

Endsley, M. R. (1995). Toward a theory of situation awareness in dynamic systems. Human Factors, 37(1), 32-64.

IATA/AEA/ERA (2013). A Blueprint for the Single European Sky.

ICAO (2012). Aviation System Block Upgrade Modules relating to Trajectory-Based Operations. Agenda item 5.2: Improved traffic synchronization through 4D trajectory-based operations (TBO). AN-Conf/ 12-WP/17, Appendix B. $12^{\text {th }}$ Air Navigation Conference.

JPDO [Joint Planning and Development Office] (2011). Targeted NextGen Capabilities for 2025.

Langan-Fox, J., Sankey, M. J. and Canty, J. M. (2009). Human factors measurement for future air traffic control systems. Human Factors, 51, 595-637.

Met Office (2014). WAFC London Performance Indicators. http://www.metoffice.gov.uk/aviation/ responsibilities/icao.

NextGen Institute (2012). Joint FAA/SESAR Technical Interchanges Report, Element 1, 2 and 3. http:/l nginstitute.org/library/documents/.

PARC/CAST Flight Deck Automation WG (2013). Operational Use of Flight Path Management Systems. http://www.faa.gov/news/fact_sheets/news_story.cfm?newsId=15434.

PRU [Performance Review Unit] (2013). ACE2011: ATM Cost-Effectiveness (ACE) 2011 Benchmarking Report with 2012-2016 outlook. Performance Review Unit. Eurocontrol.

Reynolds, T. G. and Hansman, R. J. (2003). Investigating Conformance Monitoring Issues in Air Traffic Control Using Fault Detection Approaches. Massachusetts Institute of Technology, Report No. ICAT2003-5.

Reynolds, T. G., Glina, Y., Troxel, S. W. and McPartland, M. D. (2013). Wind Information Requirements for NextGen Applications Phase 1: 4D-Trajectory Based Operations (4D-TBO). FAA-ATC-399, Massachusetts Institute of Technology Lincoln Laboratory.

RITA [Research and Innovative Technology Administration] (2014). Airline On-Time Statistics and Delay Causes; Flights: All Carriers-All Airports. U.S. Department of Transportation.

Rolfe, M. (2014). iFACTS, NATS' award winning controller productivity tool. Royal Institute of Navigation Conference. NAV Aviation: Impact of new technology and concepts on airspace capacity, safety, efficiency and cost.

Scovel, C. L. (2013). FAA's Progress and Challenges in Advancing the Next Generation Air Transportation System. Statement by Inspector General, U.S. Department of Transportation. July 17, 2013.

SESAR JU [Joint Undertaking] (2012). European ATM Master Plan. Edition 2.

Simpson, R. W. and Ausrotas, R. A. (1991). Modelling Risk in ATC Operations with Ground Intervention. Massachusetts Institute of Technology Flight Transportation Laboratory Report R91-6.

Straussberger, S. (2006). Monotony in Air Traffic Control - Contributing Factors and Mitigation Strategies. Ph.D. thesis. Eurocontrol EEC Note No. 15/06.

Welch, J. D., Andrews, J. W., Martin, B. D. and Sridhar, B. (2007). Macroscopic workload model for estimating en route sector capacity. $7^{\text {th }}$ USA/Europe Air Traffic Management R\&D Seminar.

Wickens, C. D., Li, H., Santamaria, A., Sebok, A. and Sarter, N. B. (2010). Stages and Levels of Automation: An Integrated Meta-analysis. Proceedings of The Human Factors and Ergonomics Society $54^{\text {th }}$ Annual Meeting. 